\title{
Framework for Sustaining Collaboration in Laboratory-Oriented Communities of Practice
}

\author{
Yassin Rekik, Denis Gillet, Anh Vu Nguyen-Ngoc, and Thibaud Guillaume-Gentil
}

\begin{abstract}
The paper presents a Web-based collaboration framework designed for sustaining laboratory-oriented activities carried out within academic communities of practice. This framework relies on a Web-based collaboration environment designed as an electronic notebook, such journaling resources being commonly used in laboratory activities for collecting data and thoughts, keeping analyses and notes, as well as sharing information and results. Many electronic notebook systems exist. They are however mostly domain-oriented, as the eJournal environment developed at the Ecole Polytechnique Fédérale de Lausanne (EPFL) to support collaborative experimentation in engineering education or the Collaboratories supporting experimental research in specific natural science areas. The new version of the eJournal introduced in this paper, namely the CoPs eJournal, aims at overcoming this limitation by focusing more on the context and the community. The CoPs eJournal has been developed jointly by the EPFL and the HE-Arc Ingénierie (School of engineering, University of Applied Sciences, Western Switzerland). The original environment has been designed to sustain collaboration in domains characterized by predefined roles (guests, students, assistants, and educators), predefined types of shared assets (measurement data, equipment settings, experimentation protocols or analysis scripts) and predefined privileges and services. The CoPs eJournal is designed to let the users adapting features, structures and rules according to the tacit and evolving interaction schemes driving their community. This is achieved through the definition of a community protocol. The CoP eJournal has been completely developed as a collection of Web services using the .NET Framework. It is currently refined and validated for sustaining laboratory-oriented activities into two academic contexts. The first one is a learning context where a community collaboratively exploit educational laboratory resources. The second one is a research context where a community whose members belong to different institutions jointly exploit scientific laboratory resources.
\end{abstract}

Index Terms-Collaborative work, Computer aided instruction, Educational technology, Engineering education.

Manuscript received June 4, 2006. This work was supported in part by the Board of the Swiss Federal Institutes of Technology and the European Union under the fifth and sixth framework programs.

Y. Rekik is with the School of Engineering, HE-Arc Ingénierie, University of Applied Sciences Western Switzerland, CH-2400 Le Locle, Switzerland (e-mail: yassin.rekik@he-arc.ch).

D. Gillet is with the Ecole Polytechnique Fédérale de Lausanne (EPFL), CH-1015 Lausanne, Switzerland. (e-mail: denis.gillet@epfl.ch).

A. V. Nguyen-Ngoc is with the Ecole Polytechnique Fédérale de Lausanne (EPFL), CH-1015 Lausanne, Switzerland (e-mail: anhvu.nguyenngoc@epfl.ch).

T. Guillaume-Gentil is with the Ecole Polytechnique Fédérale de Lausanne (EPFL), CH-1015 Lausanne, Switzerland (e-mail: thibaud.guillaume-gentil@epfl.ch).

\section{INTRODUCTION}

$\mathrm{T}^{\mathrm{N}}$ various fields, particularly in natural sciences and engineering education and research, laboratory resources and activities play a central role [1,2]. Over the last decade, the development of the Internet has brought new opportunities in the way students and scientists can access and exploit laboratory resources. Thanks to many initiatives, collaborative Web-based activities dealing with real or simulated equipment are becoming common and effectively assessed, as example for Web-based experimentation [3, 4]. It is now possible to access remotely laboratory resources at anytime and from anyplace, to conduct collaborative design activities with team distributed worldwide or to monitor an industrial facility at distance just to mention a few. The current trend is also to provide such services to mobile users.

In this paper, the flexible integration and collaborative usage of laboratory resources to support knowledge building and sharing within communities of practice (CoPs) is discussed. For more than ten years, CoPs have been recognized as effective environments to support learning in professional organisations and educational institutions [5]. Collaborative learning is inherent in such communities where members interact by making their knowledge and practices explicit, sharing them with their peers, and reflecting on them $[6,7]$.

CoPs are groups of people who share a concern, a set of interests, or a dedication to special topics (the domain of the community), who want to deepen their practical knowledge and expertise in the considered domain (the practice of the community), and who interact and collaborate through open and moving rules and structures (the community itself). CoPs have several characteristics that distinguish them from formal organizations. CoPs are mainly based on flat structures and driven by tacit rules, with none or weak hierarchy and none or weak institutional control.

In laboratory-oriented $\mathrm{CoPs}$, the assets produced, exchanged and manipulated by the community members can be more volatile, dynamics and rich than the typical information medias found in other communities. In addition to text documents, images, and videos, the laboratory assets include experiment-related data.

This paper proposes an innovative framework for sustaining collaboration within laboratory-oriented CoPs. This framework, namely the CoPs eJournal, has been developed as an electronic version of the classical laboratory notebook. The 
CoPs eJournal is actually an implementation of the concept of mediation artifacts [8], which was originally deployed at the EPFL as part of a Web-based experimentation environment to support hands-on engineering education. The proposed framework widens and extends this context-specific solution to support generic laboratory-oriented CoPs.

Section 2 presents the original version of the eJournal dedicated to collaborative Experimentation engineering education. Section 3 introduces the specific requirements for laboratory-oriented $\mathrm{CoPs}$ and details the extensions leading to the CoPs eJournal. Section 4 presents the implementation of the Web-based framework designed for sustaining collaboration in laboratory-oriented communities of practice. The paper concludes with perspectives and future works.

\section{Original eJournal Dedicated to Collaborative EXPERIMENTATION ENGINEERING EDUCATION}

Laboratory activities in engineering education represent a very complex socio-technical setting. They rely on the coordinated exploitation of protocols, instrumentation, laboratory equipment and analysis tools, as well as on sustained exchanges with peers, technicians, teaching assistant and educators. When laboratory activities are mediated using Web technologies to enable flexible and remote experimentation, the problem becomes even more complex. In such a case, the required Web-based solutions have to support the interaction at three different levels: (i) human-human interaction (interaction and collaboration between people), (ii) human-system interaction (manipulation of remote equipments, access to simulation tools, management and sharing of data), and (iii) system-system interaction involving exchanges between different components (for example, transferring data from an acquisition to an analysis tool).

These three levels of interaction rely on the collection and exchange of digital assets, such as measurement data, equipment settings, experimentation protocols or analysis scripts, which can be considered as mediation artifacts [9-11]. These artifacts can be adequately stored in electronic shared spaces [12]. The metaphor of paper laboratory notebooks traditionally used in laboratory activities can be use to design electronic shared spaces. The resulting electronic notebooks emulate and enhanced the functionalities and the access modes of their paper counterparts. As a mean to access and integrate the experiment-related assets, an electronic notebook can also be considered as a mediation artifact.

The mediation artifacts can be considered simultaneously as a means and a product of the interaction and collaboration process in Web-based laboratory activities. Working with artifacts can trigger new actions and can result in the modifications of existing artifacts or the creation of new ones. For example, the exploitation of an artifact corresponding to an experimentation protocol is a means to ask students to use instrumentation for acquiring measurement data that will be saved as a new artifact.

An extended electronic laboratory journal, namely the eJournal, has been developed at the Automatic Control Laboratory at the EPFL to provide students with a notebook- like electronic space for collecting and sharing assets while carrying out laboratory activities using the eMersion Webbased experimentation environment [1, 3]. As an implementation of mediation artifact, the main roles of the eJournal is to support and sustain the synchronous interaction and collaboration process among students, the online tutoring by teaching assistants, the submission of laboratory assignments and the supervision of the class progresses by the educators.

The eJournal integrates services that mediate the interaction at the three levels previously mentioned. In addition, these services are especially helpful in sustaining the continuity of interaction, providing awareness, and enabling evaluation as detailed below:

- By allowing team members or Web services to store and to access the artifacts produced and manipulated during the experimentation activities through a unique mechanism at anytime and from anyplace, the eJournal turns to be a pivotal component to maintain the work context and to avoid discontinuity of interaction. For example, there is no need for a user to send measurement data as email attachment to a colleague. Once an acquisition is completed, the measurement are automatically store within the eJournal by the acquisition service and instantaneously available to all team members, as well as to the analysis service.

- By logging and tracking all the actions performed on the artifacts by team members and the Web services within the eJournal, awareness information can be provided. Such information regarding the group and the class progress turns to be instrumental in sustaining motivation among learners, in enhancing collaboration and in supporting tutoring.

- By collecting and analysing information about artifacts production, manipulation and circulation among students, the eJournal turns to efficiently support the utility and usability evaluation of the learning modality and the learning environment. For example, studying if the assets are created on campus or from home, during the office hours or at night, can indicate if the practice is in line with the envisioned pedagogical scenario.

This first version of eJournal has been deployed and evaluated both at EPFL and at other European universities, and the results are very encouraging as presented in $[16,17]$. However, this version has been designed for a predefined community with predefined structure and rules. In order to extend the eJournal and to cover other contexts, a new model and a new design have been proposed and have led to the CoPs eJournal Framework presented in the next sections.

\section{EXTENDED EJOURNAL DEDICATED TO LABORATORY- ORIENTED COMMUNITIES OF PRACTICE}

In order to extend the eJournal so that it could support multiple contexts in various communities, a re-design of the eJournal has been performed. This new design aims at sustaining the collaboration in communities having different structures and ruled by different protocols and policies. The 
idea is to evolve from a dedicated tool to an extended and generic software framework capable of adapting its functions and services to various CoPs. Towards this goal, a model for Laboratory-Oriented Communities of Practices is first proposed. Then, appropriate software architecture is defined. These two steps are presented in the following sub-sections.

\section{A. Model for Laboratory-Oriented CoPs}

Many models of CoPs already exist [5, 18]. The model proposed here is a simplified one, which consider the main aspects of these models but allows also considering the concepts behind the original eJournal. The main features considered for defining laboratory-oriented $\mathrm{CoPs}$ are Community Structure, Community Protocol, Assets, and Activities. These four concepts are detailed in the next subsections.

\section{1) Community Structure (Members, Groups)}

The community structure represents the organisation of the community. It consists mainly of the definition of all members of the community and their repartition into groups of interests. The repartition of community members into groups of interests allows providing multiple views of the community artefacts. A personal view enables accessing and manipulating personal artefacts. A group view allows accessing and manipulating artifacts shared by group members. A community view allows to accessing and manipulating all the artifacts of the community. Of course, manipulation of artifacts produced by other members should be restricted according to the artifact properties and to the member role.

A member of a community can be involved in several groups with a different role in each group.

2) Community Protocol (Actions, Roles, Rights)

The community protocol defines all the rules governing the exchanges, the actions and the activities within the community. In the eJournal framework, it defines who can do what with which assets. So, the protocol lists the roles within the community and the rights associated to each role for performing actions on individual, group or community assets.

For example, an asset can be annotated, broadcasted, associated with an activity, finalized and submitted by members of a group of authors before being assessed by members of a group of reviewers.

3) Concept of Assets (Assets, Categories, Links)

As a mediation artifact, the eJournal integrates and enables the management of all assets produced by the community. The assets are atomic artifact produced, manipulated and exchanged between members of the community, between members and system components, and between system components themselves.

In a community, depending on its focus and interests, the assets can be grouped into different categories. The categories change from one community to another and have to be defined when a new community is created. The list of categories can also be extended dynamically during the life of the community.

In addition, assets can be associated together through semantic links. Again, the list of possible links depends on the community and has to be defined when a new community is created. It can also be extended dynamically afterwards.

By letting the community defining itself its categories and links, customized terminology and classifications suiting better its domains of interest can be chosen.

4) Concept of Activity (Activities, Tasks, Deliverables)

CoPs can range from well-structured entities having defined objectives and activities to flexible entities allowing members to interact and communicate without predefined objectives. In the case of well-structured CoPs, it is necessary to classify the actions and the work that can be performed within the community as specific activities. A community can have several running activities. Several groups can, jointly or separately, work and contribute in each of these running activities.

An activity is composed of a set of tasks to be achieved. These tasks can be sequential or parallel. For each task, it is possible to associate one or several deliverables. Deliverables are assets proposed by community members as a contribution to achieve a task. There are two kinds of deliverables: with or without review. Deliverables without review are automatically considered as completed when an authorized member associates an asset to it (we call this action an asset submission). For deliverables with review, submitted assets have to be accepted by a reviewer. The list of reviewers for a deliverables is defined through a list of roles authorized to perform the reviewing.

\section{B. Software Architecture for CoPs eJournal Framework}

The CoPs eJournal Framework is made of a central component and three other peripheral ones. The core component of the framework, namely the eJournal Server Application, is responsible of storing and managing all the information related to active communities. This application interacts with the eJournal Database and provides a set of services to answer the requests of the other components. The three other peripheral components allow the eJournal Server Application to interact with the three main actors or users for whom the Framework is dedicated: eJournal Administration Application for administrator, eJournal Client Application for community members, and Import-Export API for interaction with external services. Figure 1 illustrates the architecture of the CoP eJournal framework.

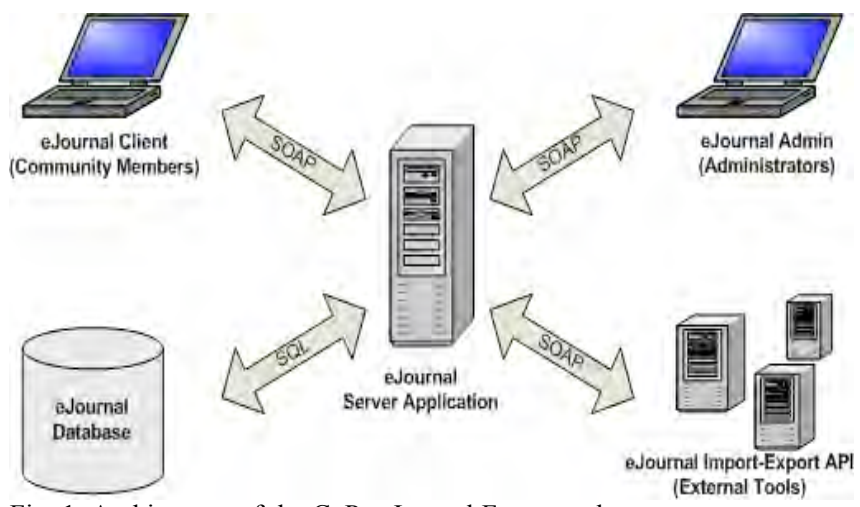

Fig. 1. Architecture of the CoPs eJournal Framework

1) eJournal Server Application

The eJournal Server Application is a set of services allowing the implementation of the Laboratory-Oriented CoPs 
model. The services provided by this component can be divided into 4 main categories:

- Members Management Services allow creating new communities, adding members, and structuring members into groups.

- Protocols Management Services allow defining possible roles for members and specifying accessible views and authorized actions for each role.

- Assets Management Services allow defining categories and links for assets when creating new communities or modifying them dynamically afterwards. They also allow members to manipulate assets through authorized actions depending on their roles and associated rights.

- Activities Management Services allow defining activities with associated tasks and deliverables. They also allow controlling activities' progress and achievement.

\section{2) eJournal Administration Application}

The eJournal Administration Application is the interface for administrators to configure and supervise the eJournal Server Application. It allows him to create new communities, to define their specific features (roles, rights, links, and categories), to manage their members and groups, and to structure their activities. The eJournal Administration Application exploit the services provided by the eJournal Server Application in order to help administrators in managing active communities and creating new ones.

It is very important to mention that some features of a community can evolve during its life. For example, members of a community can dynamically add new semantic links or new categories for manipulated assets. However, other features are definitely fixed after a community is created. For example, it is possible to add new roles in a community, but if one role is created and its rights defined, this can't be changed afterwards.

\section{3) eJournal Client Application}

The eJournal Client Application is the interface for community members to use the eJournal Server Application. It is designed as a generic application allowing community members to produce, exchange, share and manipulate artifacts in order to achieve tasks and complete activities.

The eJournal Client Application has the structure and GUI for all members of a community. However, the information it provides and the actions it allows is context and member dependent.

As for the eJournal Administration Application, the eJournal Client Application exploits the set of services provided by the eJournal Server Application in order to define the context of a user and to provide him with appropriate functions and facilities.

\section{4) eJournal Import-Export API}

One of the objectives when working on sustaining collaboration in laboratory-oriented CoPs is to enhance the continuity of interaction between the various contexts and the different stages associated with laboratory activities such as planning, preparation, experimentation or analysis. Since outputs from one context or stage may serve as inputs for another context or stage, a linkage mechanism is necessary. This mechanism has to minimize the interruption and the manipulation when switching between contexts and jumping between tasks.
As a pivotal component, the eJournal Framework enhances the continuity of interaction by providing Import-Export facilities allowing external tools to export assets to the eJournal and to query and access assets from the eJournal. Technically speaking, the API is a set of services provided by the eJournal Server Application accessible for external tools and offering documented set of facilities to import and export contextual assets to and from the eJournal Framework.

\section{IMPLEMENTATION OF THE FRAMEWORK}

Basing on the specification described above, we have implemented a prototype of the CoPs eJournal framework. This prototype has been developed using the .NET technology. All the Web Services involved in the eJournal Server Application have been coded using the $\mathrm{CH}$ language. The eJournal Client Application and the eJournal Administration Application have been developed as ASP pages. Finally, we have adopted MySQL to store and manage the data.

We have granted a particular interest to the development of the eJournal Client Application and the design of its GUI. Our objective was to satisfy three main requirements. The first requirement is that the eJournal Client has to provide a common GUI for all the community members. However, this GUI must be automatically adapted to the profile and the context of the current user. For example, actions that are not authorized for the current user must be hidden. The second requirement is to group all the display and manipulation of the eJournal Client within a common window. The idea is to avoid the complexity of switching between numbers of windows when the eJournal Client is integrated into a Webbased experimentation environment. Finally, the eJournal Client must provide GUI in multiple languages.

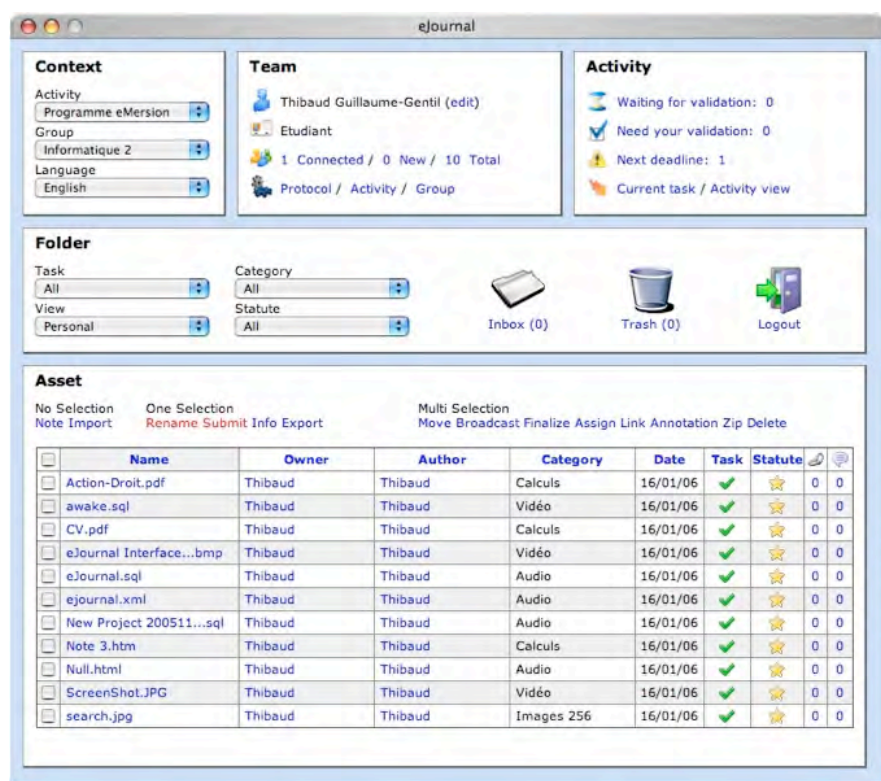

Fig. 2. The eJournal Client GUI with its main Panels

Basing on these requirements, we developed a prototype of the eJournal Client, which is made of multiple panels managed within one main window. Figure 2 shows the GUI of the eJournal Client with its involved panels. These main panels are: 
- The context panel, which allows the user to define his work's context. In this panel, the user can select the activity he wants to work on, the group he wants to join, and the language he wants to use.

- The team panel, which provides the user with synthetic information about his profile, mainly identification and role, and the profile of the group he joined, mainly online partners, new subscribed members, and so on.

- The activity panel, which provides the user with all the information related to his activities: current task, progress level, next deliverables, and so on.

- The folder zone, which allows the user to select subsets of assets using appropriate filters. The eJournal provides four filtering criteria: the task, the asset category, the asset status, and the view. Three views are provided: personal view, group view, and community view.

- The asset panel, which is the main one allowing the user to access the list of assets he owns and/or he shares, and to manipulate these assets through the set of provided functions.

The main actions that members can perform on an asset have been grouped into three categories. The first one involves actions that require a pre-selection of one ad only one asset. The second category involves actions that can be associated to a set of assets, and finally, the third category involves actions that are not associated to existing assets. Here is the exhaustive list of actions provided by the eJournal Client:

- Import allows a member to import an external asset to its personal eJournal.

- Note allows a member to create a new asset of type "note", which are html pages created within the eJournal using an integrated editor.

- Rename allows a member to rename the pre-selected asset.

- Submit allows a member to submit the pre-selected asset as a deliverable.

- Info allows a member to access all the meta-data associated to the pre-selected asset.

- Export allows a member to export the pre-selected asset to its hard disk.

- Move allows a member to move a set of pre-selected assets from its personal eJournal to other eJournals.

- Broadcast allows a member to send a set of pre-selected assets to one or to a set of other members. These broadcasted assets will be sent to the inbox of the receivers.

- Finalize allows a member to tag a set of pre-selected asset as finalized. This tag allows separating between draft assets and finalized ones.

- Assign allows a member to assign a set of pre-selected assets to a specific task within the current activity.

- Link allows a member to create a semantic link between assets.

- Annotation allows a member to add annotation and to associate them to a set of pre-selected assets.

- Zip allows a member to create an archive from a set of pre-selected assets.

- Delete allows a member to delete a set of pre-selected assets.

Depending of the context, only a subset of actions will be active. All unauthorized actions will be inactivated.

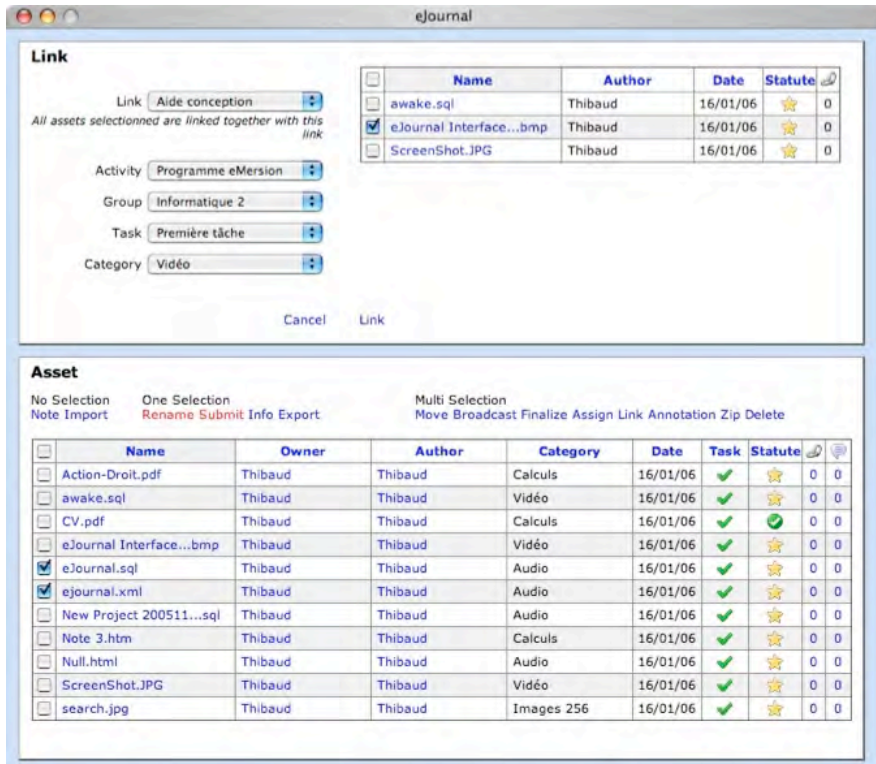

Fig. 3. Activation of the Link Action

In order to maintain all the panels of the eJournal Client within the same window, all panels associated to actions have been designed to cover the upper side of the eJournal Client. These panels hide, temporary, the context, team, activity, and folder panels. The figure 3 shows the case where a member is creating semantic links using the Link action.

\section{CONCLUSION}

In this paper, we presented a Software Framework, namely CoPs eJournal, developed jointly by the He-Arc and EPFL, with the aim of sustaining collaborative learning and knowledge building within Laboratory-Oriented Communities of Practice. Contrary to traditional learning modalities, CoPs are non-formal structures, often virtual, governed by variable rules, often built dynamically by community members. Thus, CoPs eJournal has been designed to fulfill the requirements of this specific context and to make it possible for a community to build its own organization by defining possible roles, accessible services, and shared vocabularies and semantics.

The implementation of the CoPs eJournal Framework has been based on a previous work where we had, first, proposed a model for Laboratory-Oriented CoPs and then worked on a specification for appropriate software architecture. Currently, we are validating the CoPs eJournal into two academic contexts. The first one is a learning context where a community collaboratively exploit educational laboratory resources. The second one is a research context where a community whose members belong to different institutions jointly exploit scientific laboratory resources.

In parallel with our validation work, we are currently working on some improvements and extensions. The first direction we are investigating is mobile CoPs. The idea is to develop a new eJournal Client that can be accessed through mobile devices by mobile users. This new Client is supposed to provide synthetic information, simplified views, and new kind of awareness (for example, SMS-based notification).

The second issue we are working on is to couple the CoPs eJournal with efficient knowledge management tools. The idea is to help communities to build their own ontologies and to benefit from knowledge extraction and classification services. 
The last aspect we are targeting is to enhance voice/videobased communication within CoPs. The idea is to reinforce activeness of community members by encouraging dialogue and argumentation, by increasing motivation, and by building mutual trust.

\section{REFERENCES}

[1] D. Gillet, F. Geoffroy, K. Zeramdini, A. V. Nguyen-Ngoc, Y. Rekik, and Y. Piguet, "The Cockpit, An effective Metaphor for Web-based Experimentation in Engineering Education," International Journal of Engineering Education, vol. 19, pp. 389-397, 2003.

[2] L. D. Feisel and A. J. Rosa, "The role of the laboratory in undergraduate engineering education," ASEE Journal of Engineering Education, vol. 94, 2005.

[3] D. Gillet, A. V. Nguyen-Ngoc, and Y. Rekik, "Collaborative Webbased Experimentation in Flexible engineering education," IEEE Transactions on Education, Special issue on Web-based Instruction, vol. 48, pp. 696-704, 2005.

[4] A. Böhne, N. Faltin, and B. Wagner, "Synchronous tele-tutorial support in a Remote laboratory for Process control," in Innovations 2004: World Innovations in Engineering education and research, W. Aung, R. Altenkirch, T. Cermak, R. W. King, and L. M. S. Ruiz, Eds. New York, USA: iNEER in cooperation with Begell House Publishers, 2004, pp. 317-329.

[5] E. Wenger, Community of practices: learning, meaning and identity: Cambridge University Press, 1999.

[6] P. Dillenbourg, "What do you mean by 'collaborative learning'?" in Collaborative learning: Cognitive and Computational approaches, $\mathrm{P}$. Dillenbourg, Ed.: Oxford: Elsevier, 1999, pp. 1-19.

[7] A. Fjuk, "Computer Support for Distributed collaborative learning. Exploring a complex problem area," PhD thesis. Department Informatics. University of Oslo, 1998.

[8] A. V. Nguyen-Ngoc, "Web-based Interaction and Collaboration in Flexible engineering education. An artifact-based approach." $\mathrm{PhD}$ thesis. Ecole Polytechnique Federale de Lausanne (EPFL), 2006.

[9] P. H. Carstensen, "Computer Supported Coordination," PhD thesis. Roskilde University, 1996.

[10] K. Schmidt and L. Bannon, "Taking CSCW seriously: Supporting articulation work," Computer Supported Cooperative Work (CSCW): The Journal of Collaborative Computing, vol. 1, pp. 7-40, 1992.

[11] A. G. Sellen and R. H. R. Harper, The myth of the paperless office: MIT Press, 2002.

[12] M. Robinson, "Design for unanticipated use," presented at European Conference on Computer Supported Cooperative Work (ECSCW), Milano, Italy, 1993.

[13] L. J. Bannon and S. Bødker, "Constructing Common Information Spaces," in ECSCW97: Proceedings of the 5th European CSCW Conference, ECSCW 97:Proceedings of the 5th European CSCW Conference., J. Hughes, T. Rodden, W. Prinz, and K. Schmidt, Eds.: Kluwer Academic Publishers, 1997.

[14] D. Salber, "De l'interaction individuelle aux systèmes multi-utilisateurs. L'exemple de la Communication Home-Home-Médiatisée." PhD thesis. Univesité Joseph-Fourier, 1995.

[15] S. Sire, "The direct collaboration," PhD thesis. University of Toulouse $1,2000$.

[16] A. V. Nguyen-Ngoc, D. Gillet, and S. Sire, "Evaluation of a Webbased learning environment for Hands-on experimentation," in Innovations 2004: World Innovations in Engineering Education and Research, W. Aung, R. Altenkirch, T. Cermak, R. W. King, and L. M. S. Ruiz, Eds. New York, USA: iNEER in cooperation with Begell House Publishing, 2004, pp. 303315.

[17] A. V. Nguyen-Ngoc, Y. Rekik, and D. Gillet, "Formal assessment of a Web-based learning environment," presented at iNEER Conference on Engineering Education and Research, Tainan, Taiwan, 2005.

[18] J. M. LaContora and D. J. Mendonca, "Communities of practice as learning and performance support systems," presented at International Conference on Information Technology: Research and Education, 2003. 